\title{
Development of an adaptive surface irrigation system
}

\author{
S. Shahidian $\cdot$ R. P. Serralheiro
}

Received: 11 March 2010/Accepted: 4 January 2011

(C) Springer-Verlag 2011

\begin{abstract}
Cablegation is a simple system for automating surface irrigation in small- and medium-sized fields using a gated pipe. In this work, a Programmable Logic Control, PLC, was used to develop an adaptive cablegation system capable of establishing the infiltration equation in real time and then adjusting the irrigation times to the infiltration rate and field geometry. A controlling program was developed for the on-field determination of the infiltration equation, simulation of advance in each furrow, and the optimization and management of the irrigation event. The equipment was tested in three experimental stations, including a Luvissol field organized in contour terraces with furrows of various lengths. The results demonstrate the capability of the system to adapt the application times to the different furrow lengths and the gradual decrease in the soil infiltration and to recommend an application depth that optimizes the Application Efficiency. Various improvements were made to this solar-powered cablegation, resulting in a reliable surface irrigation system capable of unsupervised operation.
\end{abstract}

\section{Introduction and objectives}

The non-renewable nature of the world's energy sources spells an inevitable increase in energy and raw-material costs, which will sooner or later question the economic feasibility of some pressurized irrigation systems. Additionally, there is a growing environmental concern with the

Communicated by T. Trooien.

S. Shahidian $(\bowtie) \cdot$ R. P. Serralheiro

Universidade de Évora, ICAAM, Apartado 94,

7000 Évora, Portugal

e-mail: shakib@uevora.pt annual waste of plastic in driplines and the emissions associated with pressurizing water. Nevertheless, the most pressing concern is probably the runoff and erosion from center pivots in soils with low infiltrability (Luz and Heermann 2005; Santos et al. 2003). This is particularly the case in southern Portugal where these systems have been installed extensively in areas with rolling hills and very shallow soils of low infiltration, such as Luvissols. The continued degradation of these soils associated with the end of subsidies to offset the installation costs provides little incentive for farmers to invest in costly irrigation systems.

Against this background, it might be timely to prepare for a potential renewed interest in alternative systems, such as surface irrigation, that can adequately handle small- and medium-sized fields. At the same time, technological advances in recent years allow the development of automated surface irrigation systems that in association with available conservative technologies can provide farmers with reliable and eco-friendly alternatives.

In surface irrigation, performance is very much dependent on the infiltration characteristics of the soil, which are not constant. They change with initial soil water content, roughness, and from one irrigation event to next. Thus, any automatic surface irrigation system must be able to foresee or measure infiltration and advance time during the early stages of the irrigation and make the necessary adjustments to application rate and depth in real time. Additionally, the actual field geometry and its organization, such as terracing, result often in furrows with different lengths, which present an added challenge, since they need to receive the same application depth.

The aim of the present work was to develop an automatic cablegation system that at the start of each irrigation adapts the application times to existing soil infiltration and individual furrow lengths. Such a system when used in 